\title{
El diseño para adultos mayores: un enfoque centrado en la persona
}

\begin{abstract}
Resumen
El presente artículo se deriva de la investigación de carácter doctoral "Diseño de un modelo para la reflexión identitaria de la persona mayor con demencia a través de la cultura material". El objetivo de evidenciar la importancia que tiene el diseño para personas mayores con un enfoque centrado en la persona, se concretó utilizando una metodología cualitativa, de carácter etnográfico, con la cual se visibilizó que la demencia acentúa la necesidades socio emocionales de las personas. Se halló información que refuerza la idea de la importancia de los objetos en la vida cotidiana de las personas mayores como elementos detonantes de memorias y testigos de vida, pues existen objetos que fungen como promotores de una reflexión de identidad, como espejos que permanecen presentes ante la persona para, de alguna manera, recordarle quien es. Se concluye que este tipo de objetos confirman que es necesario que el diseño para personas mayores vaya más allá de la función y la accesibilidad, siendo necesario ampliar la visión que en académica y profesional que se tiene respecto al diseño para adultos mayores en el contexto latinoamericano y la inclusión de aspectos sociales, culturales y emocionales es irrebatible.
\end{abstract}

\author{
Annika Maya Rivero \\ Doctorado en Diseño \\ Universidad Autónoma del Estado \\ de México \\ Toluca, México. \\ Correo electrónico: \\ amayar004@profesor.uaemex.mx \\ ๑ orcid.org/0000-0002-2648-4977 \\ Google Scholar
}

Recibido: septiembre 22 de 2018 Aprobado: junio 08 de 2020

Palabras clave: demencia, envejecimiento, gerontodiseño, identidad, personas mayores. 


\section{Design for older people: A person-centered approach}

\begin{abstract}
This article is derived from the doctoral research "Design of a model for the identity reflection of the elderly person with dementia through material culture". The objective of demonstrating the importance of design for older people with a person-centered approach was specified using a qualitative, ethnographic methodology, with which it was made visible that dementia accentuates people's socio-emotional needs. Information was found that reinforces the idea of the importance of objects in the daily life of older people as elements that trigger memories and are witnesses of life, since there are objects that act as promoters of a reflection of identity as mirrors that remain present before the person to, in some way, remind them of who they are. It is concluded that this type of objects confirm that it is necessary that design for the elderly go beyond function and accessibility, being necessary to broaden the academic and professional vision of design for the elderly in the Latin American context, and the inclusion of social, cultural and emotional aspects is irrefutable.
\end{abstract}

Key words:

dementia, aging, gerontodesign, identity, older people. 


\section{Introducción}

Como parte de una investigación doctoral enfocada en la promoción de la reflexión identitaria de las personas mayores con demencia, se realizaron exploraciones a distintos tópicos que se relacionaban con el tema. La primera aproximación al tema incluyó un año de investigación etnográfica en centros de día y residencias geriátricas en México, al efectuar la investigación de campo fue que se reafirmó que cuando se habla de diseño para personas mayores (gerontodiseño, diseño para la vejez, diseño para el envejecimiento) es común relacionarlo con la funcionalidad, la accesibilidad y los factores ergonómicos y que poco se ha pensado en los aspectos socioculturales que se requieren para dar solución a diversas problemáticas del llamado mercado sénior, sobre todo en países latinoamericanos como México.

Los profesionales de la salud, familiares y gente que se relaciona con cuestiones de envejecimiento, vejez, demencias y cuidados paliativos relacionan al diseño para personas mayores con aspectos funcionales, diseño de ayudas técnicas como andadores o bastones, mas no con la solución de otros requerimientos de índole social, emocional o cultural. Por ello, este artículo busca exponer los antecedentes y referentes de diseño para personas mayores, específicamente del diseño para personas mayores con demencia, con la finalidad de brindar un análisis que invite a la reflexión del cómo es que estamos diseñando para la gente mayor en América Latina, pues el diseño para la vejez (design for aging) en otras latitudes, tiene ya cierto peso dentro del quehacer del diseñador, algo que visiblemente en países como México no ha ocurrido.

El gerontodiseño es una aglutinación de la gerontología con el diseño en el sentido de existir un designio de proyectar, concebir y adaptar modelos a los adultos mayores y no de maliciosamente conspirar para vender modelos diseñados para personas con deficiencias. (Parra-Marujo, 2006, p. 3) 
El gerontodiseño tiene su base en el problema social, cultural, antropológico y de salud que se presenta en la actualidad. Parra Marujo (2006) nos habla de que el ser humano envejece no solo fisiológicamente, sino socialmente y esto provoca un abandono semiótico progresivo de la imagen del cuerpo, esta visión del diseño para personas mayores va mucho más allá del diseño accesible, el diseño universal e incluyente, puesto que se habla de aspectos psicosociales, así como socioculturales del ser humano, aspectos que suelen olvidarse al diseñar para personas mayores.

\begin{abstract}
El diseño para todos tiene en cuenta la diversidad humana, la inclusión social y la igualdad y tiene como objetivo hacer posible que todas las personas dispongan de igualdad de oportunidades y de participar en cada aspecto de la sociedad. Para conseguir esto, el entorno construido, los objetos cotidianos, los servicios, la cultura y la información, en resumen, todo lo que está diseñado o hecho por personas para las personas debe ser accesible, y útil para todos los miembros de la sociedad y consecuente con la continua evolución de la diversidad humana. (EIDD, 2004)
\end{abstract}

Se puede decir que el diseño universal resuelve muchos de los problemas de accesibilidad, tanto de mayores como de personas con problemas funcionales. El gerontodiseño busca, además, centrarse en la persona mayor para ofrecer soluciones que vayan más allá de la función. Abarcando aspectos socioculturales como las tradiciones, los usos y costumbres, entre muchos otros temas que hacen de la persona mayor lo que es.

\title{
Antecedentes del diseño para la vejez
}

En 1800 se inició el estudio de la relación del hombre con los efectos del ambiente relacionados con la luz, el sonido, el peso y otras variables (Kopec, 2012, p. 8), a esto se le conoce hoy en día como Environmental Psychology ${ }^{1}$, disciplina que incorpora cinco perspectivas psicológicas para analizar como el diseño del ambiente genera una respuesta en la persona. En lo que respecta 
al diseño ambiental para adultos mayores, este ha sido abordado desde hace unas décadas atrás. En los años cincuenta James E. Birren fue el iniciador de la Ilamada ecología del envejecimiento o gerontología ambiental ${ }^{2}$ (Hans-Werner Wahl y Weisman, 2003).

Fue hasta los años 70, cuando M. Powell Lawton y Lucille Nahemow (1973) definieron a la gerontología ambiental como la adaptación del hombre a su ambiente; una década después, Lawton (1989) desarrolló un marco de factores fisiológicos entre la casa y el adulto mayor, además de hablar sobre identidad, emoción y apego (12-29) y de manera casi paralela el diseñador Victor Papanek abordaba al diseño social (1984), pero a pesar del trabajo de Papanek en los años noventa se decía que el diseño retrasaba a la gerontología ambiental, ya que de acuerdo con M. Powell Lawton y Patricia Parmelee (1990), los diseñadores no generaban teoría lo cual colocaba al diseño como un área del conocimiento lejana a la posibilidad de mejorar la calidad de vida de las personas mayores, pues era menester de los gerontólogos y psicólogos esta tarea. También, paralelamente a Papanek y Lawton, Patricia Moore realizaba su épico viaje por los Estados Unidos de Norteamérica y Canadá, disfrazada de una mujer mayor de alrededor 80 años (1985), la diseñadora industrial y gerontóloga fue pionera en realizar este tipo de técnicas de investigación, que hoy en día nombramos diseño centrado en la persona.

Pasaron diez años para que se evidenciara que las intervenciones de diseño ambiental generaban diferencias significativas en las respuestas de las personas mayores con demencia (Day, Carreon \& Stump, 2000). En 2003, el arquitecto John Zeisel en conjunto con M. Powell Lawton y otros investigadores, demostraron el potencial que el ambiente tiene para mejorar los síntomas

\footnotetext{
${ }^{2}$ La gerontología ambiental es una área de conocimiento de la gerontología que tiene por objetivo conocer, analizar, modificar y optimizar la relación entre la persona que envejece y su entorno físico-social, desde perspectivas y enfoques interdisciplinarios, que abarcan disciplinas como geografía, psicología, arquitectura, diseño, urbanismo, ciencias de la salud, trabajo social, sociología y otras ciencias afines (Wahl \& Weisman, 2003; Rowles \& Bernard, 2013) citados en Sánchez González (2015).
} 
de la demencia tipo Alzheimer, a través de un balance entre el tratamiento farmacológico como el tratamiento del comportamiento por medio del medio ambiente que rodea a la persona.

\section{Diseño para personas mayores con demencia}

El diseño para personas mayores con algún tipo de demencia, como lo es el Alzheimer, nos ayuda a observar de cerca la importancia de un diseño integral que vaya más allá de la función, pues pareciera que al hablar de vejez inmediatamente el ser humano perdiera, ante los ojos del diseñador, todas las características particulares que lo conforman y se redujera a un usuario con problemas funcionales o déficits biológicos.

Existen publicaciones destacadas realizadas desde el diseño, en torno a la injerencia de este en la mejora de la calidad de vida de las personas mayores con demencia: Design for dementia ${ }^{3}(2010)$ y Design Innovations for Aging and Alzheimer's ${ }^{4}(2006)$ son ejemplos claros que provienen del Reino Unido y los Estados Unidos de Norteamérica, respectivamente. El diseño para adultos mayores se encuentra catalogado por la Asociación Americana de Psicología (APA) dentro de la división número 34, Ilamada Población y psicología ambiental (APA, 2017).

En 2009 se inauguraron las villas para la demencia, las cuales son un ejemplo de diseño ambiental enfocado en personas mayores, que además de buscar la accesibilidad, toman en cuenta los aspectos socioculturales de las personas que habitan el lugar, promoviendo la reflexión de identidad con ayuda del recuerdo. Como estas villas, se han generado propuestas similares en otras partes del mundo, tal es el caso expuesto en la conferencia anual de Alzheimer's Disease

\footnotetext{
${ }^{3}$ Diseño para la demencia.

${ }^{4}$ Innovaciones del diseño para el envejecimiento y la enfermedad de Alzheimer.
} 
International5: Town Square, an International Model: Designing a Dementia Friendly Immersive Replica 1950's Era Urban Adult Day Program Environment ${ }^{6}$ (Baker et al., 2017). Este tipo de propuestas retoman al diseño para la mejora de la calidad de vida, tanto de las personas mayores con demencia, como de sus cuidadores. Son ejemplo vivo de como el diseño de interiores, el diseño de productos y sobre todo el diseño de experiencias y servicios forman parte fundamental del desarrollo y mantenimiento de una sociedad envejeciente, dentro de la cual de acuerdo con diversas estadísticas (Alzheimer's-Association, 2015; Prince et al., 2015; Sosa, 2016) una gran mayoría de personas mayores desarrollarán algún tipo de demencia.

La demencia nos ha otorgado la oportunidad de darnos cuenta de que la gente mayor requiere de elementos formales que hablen de significados culturales, tradiciones, costumbres y apegos, además de la ya conocida accesibilidad. Al diseñar para personas mayores con demencia se muestran de manera evidente los rasgos de personalidad y la gran diversidad de la población adulta mayor.

Quienes se sumerjan en el diseño de productos, servicios, ambientes o experiencias para este sector se darán cuenta de la suma complejidad que implica diseñar para personas mayores, puesto que al hablar de demencia hablamos de pérdida de identidad, no solo de pérdida de funciones corporales. Entonces, si se busca diseñar para mejorar la calidad de vida de la persona mayor con demencia, debemos de introducirnos en la identidad de la persona mayor. La identidad de un ser humano no recae solamente en elementos biológicos y funcionales.

Cuando una persona presenta Alzheimer el diseño de productos puede ser similar en un inicio al diseño para personas con capacidades diferentes.

\footnotetext{
${ }^{5}$ Asociación internacional que se enfoca en investigación, difusión y soporte en torno a la demencia tipo Alzheimer.

${ }^{6}$ Town Square, un modelo internacional: Diseñando un ambiente réplica inmersivo de la era urbana de 1950, amigable con la demencia para cuidados de día para adultos.
} 
Como ejemplo tenemos a los sensores con alarmas contra incendios, colocados en las estufas o cocinas de los usuarios, dichas alarmas tienen el objetivo de evitar accidentes. Pero conforme la enfermedad progresa, los aspectos funcionales permanecen de una manera obligada, pero se muestran insuficientes ante la complejidad de la enfermedad.

En varias partes del mundo muchos diseñadores se han iniciado en el diseño para la demencia, como Adriana Machado (2015), quién desarrolla objetos personales enfocados en estimulación sensorial. Paul Rodgers, quien en conjunto con Alzheimer's Scotland, promovió que la gente recién diagnosticada con demencia trabajara en diseños de su propio tartán (tela escocesa), con el fin de eliminar el estigma que la persona con demencia adquiere al ser diagnosticada al tiempo que refuerza su identidad con el uso de lo más representativo de Escocia (2015). En el Reino Unido, existe el trabajo enfocado en la creación de objetos personales con los Ilamados sensor e-textiles, que son textiles con tecnología electrónica, esto con el fin de generar estímulos sensoriales que relajen a las personas con demencia (Treadaway \& Kenning, 2016).

El design for aging ${ }^{7}$ en países desarrollados es una temática que forma parte de estudios avanzados, existen escuelas de diseño que están totalmente enfocadas a abordar estos temas como la Universidad de Stirling (2013), donde se imparten cursos especializados en diseño para personas mayores con demencia.

\section{Método}

Mediante el diseño centrado en el usuario y herramientas etnográficas fue que se realizó trabajo de campo en una residencia geriátrica para personas mayores con demencia y en un centro de día para mayores con demencia, ambos ubicados en el Estado de México, México. La investigación de campo 
ocurrió durante un año y tuvo como objetivo realizar una exploración de cómo se imparte la terapia de reminiscencia ${ }^{8}$ a los usuarios de estos servicios, para así poder dar una respuesta desde el diseño de experiencias.

En primera instancia, se realizó observación no participante durante las primeras visitas a los centros, cada una de estas visitas con duración aproximada de una hora. Posteriormente, conforme el conocimiento relacionado con las personas mayores con demencia fue incrementado, es que la técnica de observación no participante derivó en observación participante, la cual permitió una interacción profunda con las personas, realizando bailes, cantos, juegos y pláticas con ellas. La toma de fotografías y videos no estaba permitida, así que todo se asentó en grabaciones de audio y notas en bitácora.

De manera paralela al trabajo de campo se realizó una revisión de la literatura referente al tema de demencias. En un inicio la información documental giraba en torno a los diferentes tipos de demencias que existen, su tratamiento y características particulares, luego la revisión fue más específica, profundizando en la identidad y la memoria en las personas con dicho padecimiento. La revisión tuvo el objetivo de brindar conocimiento del tema para así poder efectuar el trabajo etnográfico, mas no fue parte central de la investigación. La investigación etnográfica se nutrió visitando y analizando con base en el diseño centrado en el usuario a otros centros de día y residencias geriátricas ubicados en distintas partes de México, como: Hermosillo, Sonora; Atlacomulco, Estado de México; Ixtapan de la Sal, Estado de México y Ciudad de México. Pudiendo así convivir con más de 30 personas mayores con demencia durante el lapso de un año.

${ }^{8}$ Es una de las intervenciones psicosociales más populares, se basa en la discusión y evocación de actividades pasadas ya sea con otra persona o en grupo utilizando materiales de soporte (Cotelli et al., 2012, p. 203). 


\section{Hallazgos}

En todos los centros visitados las cuidadoras hacían referencia a la profesión de las personas a quienes cuidaban: el licenciado, el ingeniero, la maestra, el maestro, el arquitecto, entre otras. Para quienes no tenían formación académica se les decía Don o Doña. Se observó cómo las cuidadoras hacían referencia a las ocupaciones de las personas mayores, de los distintos roles sociales que cada persona vivió. Cada persona observada durante la investigación de campo solía representarse, actuar como debía actuar en su ocupación de juventud, se observó que cuando la enfermedad avanza es cuando el objeto se convierte en la única ventana de comunicación y de recuerdo.

De acuerdo con Jean Baudrillard (1969), en los objetos desaparecen muchas neurosis, se recogen tensiones y energías en duelo, es lo que les da un alma, lo que hace que sean nuestros. Esas tensiones y neurosis son las que reflejan objetos cotidianos como la franela (para el chofer), el interfón (para el licenciado) y la bolsa (para la contadora). Con base en estas observaciones provenientes del trabajo etnográfico realizado y con base en la literatura (Ash, 1996; Buse \& Twigg, 2014, 2015; Hockey, Dilley, Robinson \& Sherlock, 2013; Twigg \& Buse, 2013) es posible decir que existen objetos que fungen como promotores de una reflexión de identidad, como espejos que permanecen presentes ante la persona para de alguna manera recordarle quien es. Este tipo de objetos (sobre todo indumentaria y objetos de uso personal) confirman que es necesario que el diseño para personas mayores vaya más allá de la función y la accesibilidad.

El diseño es un acto social. El diseño del ambiente puede afectar la identidad de una persona, digamos que lo que el diseñador proporcione serán las herramientas que la persona mayor tenga para expresarse, así que nos encontramos en terrenos lejanos al diseño de ortopedia geriátrica o ayudas 
técnicas, que si bien son elementales para la mejor calidad de vida de muchos mayores, no serán la solución idílica que los diseñadores solemos proponer al hablar de diseño para personas mayores.

Por supuesto que no todos los gerontes presentan algún tipo de demencia, pero esto no es impedimento para dejar de ver la importancia emocional que ciertos elementos ambientales tienen en la vida de la gente. Haber presenciado cómo las personas mayores con demencia buscan la permanencia de su identidad a través de los objetos personales y colectivos arroja que el cambio de paradigma que ronda al diseño de espacios, productos, experiencias y servicios para personas mayores debe efectuarse, al hablar de diseño para personas mayores se debe de diseñar más allá de la accesibilidad, pues en esta etapa de la vida ocurren procesos reflexivos que son necesarios para la reconfiguración de la identidad personal, estos procesos ocurren también a lo largo de nuestra vida, pero la manera en cómo suceden en la vejez es más profunda (SalazarVillanea, 2015).

La conformación de la identidad de cada ser humano se distingue por características particulares, su estilo de vida, sus hábitos de consumo, sus redes personales y por el conjunto de objetos entrañables que posee, así como por su biografía personal, las actitudes y las capacidades así como las tendencias y hábitos personales también conforman la identidad del individuo. (Giménez, 2010)

Lo anterior pudo constatarse al interactuar con las personas en los centros, se observó como todos, aunque tuvieran problemas derivados de las demencias, presentaban una personalidad reafirmada por sus objetos personales, pudiendo ser estos: un labial, unos tenis o zapatos, aretes, bolsos o incluso gafas.

Los diseñadores, al conocer sobre el envejecimiento y la vejez humana, abrimos los ojos ante las posibilidades que existen para intervenir y dejamos en un plano casi lógico y obvio al diseño de ayudas técnicas, nos vemos en la necesidad de aplicar un verdadero diseño centrado en el usuario al proponer 
un producto, espacio o servicio para la persona mayor, que probablemente requiera algún tipo de ayuda funcional pero siempre necesitará algo que le signifique, con lo que pueda reflejarse y mantenerse vigente.

\section{Importancia del diseño para personas mayores}

Han pasado casi 20 años desde los primeros hallazgos referentes al papel que el diseño tiene en la vida las personas de edad, pero hoy en día en América Latina son pocas las universidades que instruyen a los diseñadores en diseño para la vejez. La Universidad Nacional Autónoma de México coloca en el plan de estudios de diseño industrial un apartado llamado "diseño de dispositivos para adultos mayores" dentro de un área optativa llamada "diseño para la salud" en dónde se indica que durante el semestre se llevarán dos horas a la semana de teoría relacionada con este tema (UNAM, 2017). En la Universidad Nacional de Colombia se introdujo un proyecto piloto sobre gerontodiseño y ergonomía (UNAL, 2017) de igual manera en La Habana, Cuba, se gestó un proyecto de gerontodiseño (ONDI, 2017).

Los proyectos mencionados (UNAM, UNAL y ONDI) están basados en esa área más conocida del diseño para el envejecimiento y la vejez: la accesibilidad. Es de aplaudir y celebrar estas iniciativas, pero de acuerdo con las proyecciones demográficas, no es suficiente. El papel del diseñador como generador de cuidados paliativos, de ambientes estimulantes y como generador de soluciones ante el envejecimiento poblacional son de las facetas menos conocidas o explotadas, tanto en México, como en muchos países de América Latina.

¿Será que el diseño para personas mayores en países latinoamericanos no tiene importancia, debido a que nuestra población no está tan envejecida como en otros países? Una persona mayor es quien rebasa la esperanza de vida al nacer (Beard et al., 2015) así que en unos años al hablar de "adultos mayores", 
en el caso de México, estaremos hablando de personas que rebasan los 77 años, grupo etario que será mucho más grande de lo que es al día de hoy.

\begin{abstract}
En América Latina el peso relativo de la población de 60 y más años es más acusado en las áreas rurales, vinculado a la fuerte emigración. También, se constata la existencia de una mayor vulnerabilidad de los adultos mayores en las zonas rurales que en las zonas urbanas, que afecta sobre todo a mujeres y personas de 75 y más años, debido a sus bajos niveles educativos (analfabetismo), bajos ingresos (pobreza), aislamiento, precariedad de la vivienda, problemas de acceso a los servicios sociales y de salud, limitación en transportes públicos, así como la ausencia de políticas públicas enfocadas a este colectivo. (Sánchez González, 2015, p. 99)
\end{abstract}

De acuerdo con datos de la CEPAL (Comisión Económica para América Latina y el Caribe) (2015) en Argentina la población de personas mayores de 60 años abarca alrededor del $17 \%$ de su población total y de igual manera ocurre en Colombia, donde alrededor del $17 \%$ de su población total es de personas mayores. Chile se encuentra bastante similar con un 16\%, en México este porcentaje ronda el $10 \%$ mientras que en Cuba el 20\% de la población total es mayor de 60 años. Estos datos fundamentan una nueva perspectiva del diseño ante el envejecimiento mundial y dejan claro que Latinoamérica y el Caribe han ingresado a un cambio demográfico que apunta a tener mayoría de personas mayores que de jóvenes y niños.

En 2016, México contaba con más de nueve millones de personas mayores que no requerían ayudas técnicas o cuestiones similares (INEGI, 2016). Lo anterior nos incita a reafirmar que existe una diferencia entre ser persona mayor a ser una persona con alguna capacidad disminuida, claro que el envejecimiento humano trae irremediablemente cambios funcionales, pero al darnos cuenta de que en general el adulto mayor mexicano es en su mayoría autónomo nos forzamos a dar respuestas a las preguntas: ¿qué diseñar para las personas mayores?, ¿realmente qué es el diseño para persona mayores? Bien lo dijo el Dr. Parra Marujo: "No es diseñar para personas con deficiencias" (2006, p. 3). 
Digamos entonces que la importancia del diseño el mercado sénior (tomando como referencia a los datos mexicanos) radica en un $25 \%$ de la solución de problemas funcionales y de accesibilidad y el otro $75 \%$ es lo que le da realmente peso al saber cómo diseñar para gente mayor. Ese $75 \%$ por ciento engloba a los aspectos de identidad cultural, las costumbres, las tradiciones, aspectos emocionales, la independencia económica, aspectos laborales, indumentaria, experiencias, acceso a la tecnología y si nos enfocamos en el ocio y la nueva segunda mitad de la vida que la jubilación brinda a muchos mayores, entonces hablaremos de todas las opciones de esparcimiento, recreación y salud que puedan ser de interés para este sector poblacional. Nos empezamos a dar cuenta de que nos falta mucho conocimiento sobre este grupo de gente en particular, porque al hablar de persona mayor nos hemos ceñido a decir "60 y más" algo que es completamente erróneo, ya que una persona de 60 años hoy está distante de comportarse y tener las necesidades de alguien de más de 80 años o un centenario. Conocer al adulto mayor de hoy es el primer paso para un gerontodiseño exitoso. Si no lo conocemos tenemos un problema enorme, ya que sabemos que la innovación en productos y servicios forma parte elemental de los ciclos económicos actuales, captar la atención del mercado de los mayores es necesario para mantener viva a la economía, pero también es necesario proveerles de oportunidades que les otorguen mayor autonomía e independencia, tanto funcional como económica.

Procter and Gamble ha identificado a los mayores de 50 como su público objetivo, de hecho en palabras de Sami Kahale directora de PyG del sur de Europa "acercarse a los sénior es vital para las empresas, aunque a la vez es es un reto" (Delle-Femmine, 2016) y este reto es el que se da por el desconocimiento del usuario. 


\section{Conclusión}

El diseño centrado en el usuario aplica perfectamente al hablar de diseño para gente mayor, pero para efectuar esta tarea de manera óptima es necesario dar un nombre al proceso y tener ciertas pautas a considerar, los diseñadores deben de apoyarse en la gerontología ambiental, en el gerontodiseño y así iniciar el proyecto a desarrollar. Pero al obtener un resultado es necesario no ceñirlo a esta categoría y colocarlo dentro de una visión incluyente. El diseño para personas mayores lo que menos quiere es excluir y resaltar diferencias, aunque estas existan por el simple hecho de la diversidad, el objetivo central es colaborar en la mejora de la calidad de vida de los que en pocos años serán mayoría en varias partes del mundo.

Ya lo han dicho investigadores como Sánchez González (2015), estamos muy atrasados en América Latina en lo que respecta a la inclusión académica de temas relacionados con la gerontología ambiental. Es necesario que en las universidades este tipo de materias enfocadas en diseño para personas mayores sean incluidas, no como una materia optativa, puesto que no es algo que podamos elegir, el mundo está envejeciendo y las propuestas de diseño, desde una visión positivista, no van a dar la solución requerida. Es tiempo de aceptar que el mercado está cambiando y es necesario conocerlo para poder ofrecerle, tanto soluciones funcionales y accesibles, como un gran número de propuestas que abarquen las distintas áreas de diseño, como mobiliario, indumentaria y textiles, aplicaciones para dispositivos móviles, empaques, espacios y ambientes y sobre todo poder aplicar el pensamiento de diseño (design thinking) ante los diversos retos que el envejecimiento poblacional conlleva. 


\section{Referencias}

Alzheimer's-Association. (2015). Alzheimer's disease facts and figures. 11(3), 88. Recuperado de https://www.alz.org/facts/downloads/facts_figures_2015.pdf

APA. (2017). APA div. 34: Society for environmental, population and conservation psychology. Recuperado de http://www.apadivisions.org/ division-34/index.aspx

Ash, J. (1996). Memory and objects. In P. Kirkham (Ed.), The gendered object (pp. 219-224): Manchester University Press.

Baker, M., Trde, S., Patel, S. L., Badre, K. R., Lagervall, J. A., \& Lanuzox, J. (2017). Town square, an international model: Designing a dementia friendly immersive replica 1950's era urban adult day program environment Abstract Book. Together towards a new era. Kyoto Japon: ADI.

Baudrillard, J. (1969). El sistema de los objetos. Ciudad de México, México, Siglo XXI.

Beard, J., Officer, A., Cassels, A., Bustreo, F., Worning, A. M. y Asamoa-Baah, A. (2015). Informe mundial sobre el envejecimiento y la salud (30 de septiembre de 2015 ed., pp. 266). E.U.A.: OMS.

Brawley, E. C. (2006). Design innovations for aging and Alzheimer's (1 ed.). New Jersey, EUA: John Wiley y Son, Inc.

Buse, C., \& Twigg, J. (2014). Women with dementia and their handbags: Negotiating identity, privacy and 'home' through material culture. Journal of Aging Studies, (30), 14-22. DOI: http://dx.doi.org/10.1016/j. jaging.2014.03.002 
Buse, C., \& Twigg, J. (2015). Materialising memories: Exploring the stories of people with dementia through dress. Ageing and Society. DOI: https://doi. org/10.1017/S0144686X15000185

CEPAL. (2015). Perfil nacional socio-demográfico de países en América Latina. Recuperado de http://estadisticas.cepal.org/cepalstat/Perfil_Nacional_ Social.html?pais=ARGyidioma $=$ spanish

Cotelli, M., Manenti, R. \& Orazio, Z. (2012). Reminiscence therapy in dementia: A review. Maturitas, 72, 203-205.DOI: https://doi.org/10.1016/j. maturitas.2012.04.008

Day, K., Carreon, D., \& Stump, C. (2000). The therapeutic design of environments for people with dementia: A review of the empirical research. The Gerontologist, 40(4), 397-416. DOI: https://doi.org/10.1093/ geront/40.4.397

Delle-Femmine, L. (2016, 30/05/2016). ¿Cómo compran los mayores de 50 años? A la caza del 'consumidor senior'. El País, edición Europa.

Dementia Village Architects. (2015). Villas para la demencia. Recuperado de http://dementiavillage.com

DSDC. (2013). Improving the design of housing to assist people with dementia: The dementia Service Development Centre, University of Stirling.

EIDD. (2004). Stockholm Declaration por el European Institute for Design and Disability. Recuperado de http://www.designforalleurope.org/Design-forAll/EIDD- Documents/Stockholm-Declaration

Giménez, G. (2010). Cultura, identidad y procesos de individualización. Conceptos y fenómenos fundamentales de nuestro tiempo. Recuperado de http://conceptos.sociales.unam.mx/conceptos_final/625trabajo.pdf?PHPSE $\mathrm{SSID}=\mathrm{a} 2 \mathrm{c} 966 \mathrm{a} 8 \mathrm{fe} 8 \mathrm{efdcba3f365f98e8b9225}$ 
Hans-Werner Wahl, \& Weisman, G. D. (2003). Environmental gerontology at the beginning of the new millennium: Reflections on its historical, empirical, and theoretical development. The Gerontologist, 43(5), 616-627. DOI: https://doi.org/10.1093/geront/43.5.616

Hockey, J., Dilley, R., Robinson, V., \& Sherlock, A. (2013). Worn shoes: Identity, memory and footwear. Sociological Research Online, 18(1), 20. DOI: https://doi.org/10.5153/sro.2897

INEGI. (2016). Estadísticas a propósito del día internacional de las personas de edad (1 de octubre). Aguascalientes. Recuperado de http://www.inegi.org. mx/saladeprensa/aproposito/2016/edad2016_0.pdf.

Kopec, D. (2012). Environmental Psychology for Design (O. T. Kontzias Ed.). Canadá: Fairchild Books, Inc.

Lawton, M. P. (1989). Home as an instrument of well-being in older people. En reunión anual de la Asociación de Psicólogos de Canadá. Halifax, Nueva Escocia. Recuperado de https://mayoresdehoy.files.wordpress. com/2017/05/home_as_an_instrument_of_wellbeing_in_older_people.pdf

Lawton, M. P., \& Nahemow, L. (1973). Ecology and the aging process. In C. Eisdorfer (Ed.), The psychology of adult development and aging (Vol. vi, pp. 619-674). Washington DC, EUA: American Psychological Association.

Machado, A. (2015). Diseño sensorial. Recuperado de http://adrianamachado. com

Moore, P., \& Paul, C. (1985). Disguised: A true story: W Publishing Group.

ONDI. (2017). Proyecto gerontodiseño. Recuperado de http://www.ondi.cu/ proyecto/proyecto-gerontodiseno/

Papanek, V. (1984). Design for the real world. Human ecology and social change. Londres: Thames and Hudson. 
Parmelee, P. A., \& Lawton, M. P. (1990). The design of special environment for the aged. In J.E.B. \& K.W. Schaire (Ed.), Handbook of the psychology of aging (pp. 465-489). New York.

Parra-Marujo, J. (2006). Gerontodesign: A marca de água do design, do design ergoómico, da marca ou das marcas, branca. Recuperado de JMarujo. Artician website: http://jmarujo.artician.com/portfolio/gerontodesign/

Prince, M., Wimo, A., Guerchet, M. A., Gemma-Claire., Wu., Y.-T., \& Prina, M. (2015). The global impact of dementia. Recuperado de http://www.alz. co.uk/research/WorldAlzheimerReport2015.pdf

Rodgers, P. (2015). Designing with people living with dementia. Paper presented at the Third European Conference on Design4 Health, Sheffield.

Rowles, G. (1978). Prisoners of space? Exploring the geographical experience of older people boulder colorado. Westview Press, 1, 301-314.

Salazar-Villanea, M. (2015). Aproximaciones desde la gerontología narrativa: La memoria autobiográfica como recurso para el desarrollo de la identidad en la vejez. Anales en Gerontología (7), 1-28.

Sánchez González, D. (2015). Ambiente físico-social y envejecimiento de la población desde la gerontología ambiental y geografía: Implicaciones socioespaciales en América latina. Revista de geografía Norte Grande, 97-114. DOI: http://dx.doi.org/10.4067/S0718-34022015000100006

Sosa, A. L. (2016). Ponencia presentada en el XXI Congreso nacional de Alzheimer. Información epidemiológica de las demencias en México, León Guanajuato México.

Timlin, G., \& Rysenbry, N. (2010). Design for dementia: Improving dining and bedroom $(1$ ed.). Londres, Inglaterra: Helen Hamlyn Centre, Royal College of Art. 
Treadaway, C., \& Kenning, G. (2016). Sensor e-textiles: Person centered co-design for people with late stage dementia. Working with Older People, 20(2), 86-93. DOI: https://doi.org/10.1108/WWOP-09-2015-0022

Twigg, J., \& Buse, C. (2013). Dress, dementia and the embodiment of identity. Dementia, 12(3), 326-336. DOI: https://doi.org/10.1177/1471301213476504

UNAL. (2017). Aplicación del geronto-diseño y la ergonomía en el desarrollo de productos. Recuperado de http://www.hermes.unal.edu.co/pages/ Consultas/Proyecto.xhtml?idProyecto $=35020$

UNAM. (2017). Plan de estudios licenciatura de diseño industrial. Recuperado de http://arquitectura.unam.mx/uploads/8/1/1/0/8110907/cidi-pe-2017tomo-2-vf.pdf

Zeisel, J., Nina M. Silverstein, Hyde, J., Levkoff, S., Lawton, M. P., \& Holmes, W. (2003). Environmental correlates to behavioral health outcomes in Alzheimer's special care units. The Gerontologist, 43(5), 697-711. DOI: https://doi.org/10.1093/geront/43.5.697

Como citar: Maya-Rivero, A. (2020). El diseño para adultos mayores: un enfoque centrado en la persona. Revista KEPES, 17(22), 141-160. https://doi.org/10.17151/kepes.2020.17.22.6 